\title{
COMPUTATION OF WAITING TIME TO SUCCESSFUL EXPERIMENT USING AGE-DEPENDENT BRANCHING MODEL
}

\author{
MAROUSSIA SLAVTCHOVA-BOJKOVA \\ Department of Probability and Statistics, Institute of Mathematics and Informatics, Bul- \\ garian Academy of Sciences, 8, G. Bontchev str., 1113 Sofia, (Bulgaria)
}

\section{ABSTRACT}

Slavtchova-Bojkova, M., 1999. Computation of waiting time to successful experiment using age-dependent branching model. Ecol. Modelling,

This paper considers the properties of the waiting time to survive forever of the supercritical age-dependent branching process modified with an immigration component. Conditioning on ultimate extinction of the process we analyze the conditional distribution of the cycle length and its expectation. We then derive and give estimates of the conditional expected total progeny of a cycle and its higher moments. In general, the model originates from the problem of estimating the waiting time to a successful experiment in industrial wasted water treatment by bacterial culture systems. Computer runs were made for two reproduction laws and different reproduction means. 


\section{INTRODUCTION}

The goal of the paper is to explain some puzzling phenomena and answer questions of interest for biotechnologists and environmentalists. First, how long does take the final establishment of bacterial cultures in wastewater laboratory experiments? As there is always a positive probability of extinction, it is possible to have several unsuccessful trials before the bacterial cultures start to grow irreversibly. Secondly, what conclusions can one draw from an early extinction of a bacterial culture in different types of wastewater? Does it imply that the offspring mean in these environments is low? Similarly, our study might help decision makers to take a choice based on comparative laboratory results in one and the same environment cultivated with different bacterial strains. In general, such questions related to real world problems of industrial wastewater treatment are hard to answer. One of the major reasons for this difficulty is that the circumstances under which the experiments are made in natural and artificial basins, like lagoons, ponds and lakes, are not always the same.

We present an age-dependent branching model with immigration and theoretically analyze how one can extract exact information about some important characteristics of this model, as the mean reproduction and total progeny. On the other hand, using that model, we treat the problem of inference from expected waiting times and expected progeny on the fertility rates.

We would like to point out that in the discrete-time case, the problem concerning the total progeny was investigated by several authors ( Jagers, 1975, p. 39; Harris, 1989, p. 32), however, their main interest is focussed on questions of rates of growth, and this conditioned on survival. Karlin and Tavaré (1982) studied the asymptotic behavior of the 
probabilities of hitting the absorbing states, the times needed to hit these states, and the conditional distributions of the number of particles (for models allowing catastrophes). Bhattacharjee (1987) and Shaked and Shantikumar (1987) investigated the distribution of the extinction moment for subcritical and critical Bienaymé-Galton-Watson processes. In the work of Jacobson (1985) was carried out a numerical investigation of the rate of convergence of the extinction probability for a discrete-time age-dependent branching process, in case when the life-time has a distribution function close to gamma one. Jacobson established a quick convergence for the processes that differ essentially from the critical ones. Otherwise (for critical processes) the convergence is slow. A nice extensive survey of the recent results in the theory of branching processes is given by Vatutin and Zubkov (1993).

The problem of inference from expected waiting times and expected progeny on fertility rates, was first treated by Bruss and Slavtchova-Bojkova (1999). In their article the simple case, in which all newly introduced populations are supposed to behave like independent identically distributed (i.i.d.) Bienaymé-Galton-Watson branching processes was studied. In this paper, we consider continuous-time age-dependent branching processs. Such processes in general are not Markovian. However, the results remain valid in this case, and, we are aware that this is still a simplification of a real life problem but an explicit solution can be given in terms of generating functions.

For the above mentioned theoretical results we show a computation procedure and computer runs based on binary and Poisson reproductive rule. Since Powell (1955) found that the lifespan of bacteria follows a gamma distribution, and reproduction at death is characteristic of bacteria-like organisms, a discretized gamma density was used for all runs. 
We discuss an example of "extinction bias" which may mislead decision makers in cultivation experiments.

\section{MODEL FORMULATION}

We will first outline an age-dependent branching process with immigration in the state zero. Consider a population process starting at time 0 with a single progenitor of age 0 whose life-length $\tau$ has distribution $G(t)=P(\tau \leq t), G\left(0^{+}\right)=0$. With probability $p_{k}$, $k \geq 0$ it produces at the end of its life $k$ similar individuals (of age 0 , with the same life-length $\tau$ and reproduction distribution $\left.\left\{p_{k}\right\}, \sum_{k=0}^{\infty} p_{k}=1\right)$. The probability generating function (p.g.f.) of the number of $\xi$ offspring is denoted by

$$
f(s)=\sum_{k=0}^{\infty} p_{k} s^{k}, \quad|s| \leq 1, \quad p_{k}=P(\xi=k) .
$$

Provided that there is at least one offspring, the death-and-reproduction process is repeated, and continues as long as individuals exist.

Let $\tilde{Z}(t)$ be the number of individuals existing at time $t$, or the state of the process $(\tilde{Z}(t))_{t \geq 0}$ at time $t$. Note that a line becomes extinct once $\tilde{Z}(t)=0$ for some $t$ (and for all $t$ thereafter), and that the above process is "age-dependent" (i.e. the probability that an individual living at time $t$ dies in the interval $(t, t+d t)$ is, in general, a nonconstant function of $t$ ). The process $(\tilde{Z}(t))$ is the so-called Bellman-Harris branching process (see, for example, Athreya and Ney, 1972, pp. 137-144). Every time the process $(\tilde{Z}(t))$ hits the state zero we suppose to have an immigration of one particle from an outside source. With $(Z(t))_{t \geq 0}$ we shall denote the process with immigration in the state zero.

The discrete version of such type of processes was first studied (in the critical case) by Foster (1971) and Pakes $(1971,1975)$. 
Life cycles

For a branching process with immigration $(Z(t))_{t \geq 0}$ we call life periods (cycles) the intervals $\left(t_{0}, t_{0}+T\right)$ of maximal length on which $\inf _{t_{0} \leq t \leq t_{0}+T} Z(t)>0$. Thus $(Z(t))$ may have several life-periods, the last one always being infinite, provided the process is supercritical. If the process is subcritical it will have a.s. infinitely many life periods.

\section{Total waiting time}

We are interested in the last instant $M$ of immigration, i. e. in the "birth time" of that process which will finally survive forever. Specifically, we shall derive the conditional distribution of the length $T$ of the first life period and the conditional expectation of $T$, both conditioned on the event $\{T<\infty\}$. We shall also study the expectation of the total waiting time $M$ to the beginning of the first process which will survive forever. Finally, we shall analyze the total progeny of a cycle and shall obtain its conditional expectation and variance, both conditioned on $\{T<\infty\}$.

\section{Criticality and extinction}

It is well-known from the theory of branching processes (see e. g. Athreya and Ney, 1972, pp. 139-144) that the probability $q$ of eventual extinction of Bellman-Harris process $(\tilde{Z}(t))$ is the smallest non-negative root of the equation $f(s)=s$, and $q=1 \Longleftrightarrow$ $m=f^{\prime}(1) \leq 1$. The parameter $m$ is called the reproduction mean, and the supercritical, critical and subcritical cases correspond to the relations $m>1, m=1$ and $m<1$, respectively.

So, if $Z(0)=1$ we have $P\{M=0\}=P\{$ the initial particle does not die out without any offspring $\}=1-q$. 


\section{RESULTS}

Waiting times and life-cycles to successful experiment

Let $T_{1}, T_{2}, \ldots$ be the lengths of the life periods of those consecutive processes dying out before the surviving process is initiated (i. i. d. copies of the r. v. T).

Let $v(t)=P(T \leq t)$.

The following result shows the computation of the conditional distribution of the length of a life-cycle, its conditional expectation (given extinction) and the expectation of the total length of unsuccessful life-cycles. The length $T$ of the life-period and the last instant $M$ of immigration of the process $(Z(t))$ have the following properties:

$$
P(T \leq t \mid T<\infty)=\frac{v(t)}{q}
$$

$t>0, v(0)=P(Z(0)=0)=0$, where $q$ is the extinction probability of of the BellmanHarris process $(\tilde{Z}(t))$;

$$
\begin{gathered}
E(T \mid T<\infty)=\frac{1}{q} \int_{0}^{\infty}(q-v(t)) d t \\
E(M)=\frac{q}{(1-q)} E(T \mid T<\infty)
\end{gathered}
$$

The proofs of (2) and (3) are given in the Appendix.

The total progeny of a cycle

Our particular interest will be aimed to the total progeny $N(t)$ of a cycle conditioned on the event $\{T \leq t\}$. So, let $g_{t}(s)=E\left(s^{N(t)} \mid T \leq t\right)$. 
The probability generating function $g_{t}(s)$ of the conditional total progeny given the finiteness of the first life cycle satisfies the recurrence relation

$$
g_{t}(s)=\frac{s}{v(t)} \int_{0}^{t} f\left(v(t-y) g_{t-y}(s)\right) d G(y)
$$

(See Appendix for proof.)

Now for the expected total progeny $N(t)$ of a cycle conditioned on the event $\{T \leq t\}$ we have the following properties:

$$
\begin{aligned}
m(t)=E(N(t) \mid T \leq t) & =\frac{1}{v(t)} \int_{0}^{t} m(t-y) v(t-y) f^{\prime}(v(t-y)) d G(y) \\
& +\frac{1}{v(t)} \int_{0}^{t} f(v(t-y)) d G(y)
\end{aligned}
$$

In the case $m \neq 1$ (i. e. non-critical cases) for the estimated total progeny of a cycle we have

$$
E\left(N_{\infty} \mid T<\infty\right)=\frac{1}{1-f^{\prime}(q)}
$$

Its variance is as follows

$$
\operatorname{Var}\left(N_{\infty} \mid T<\infty\right)=\frac{1}{\left(1-f^{\prime}(q)\right)^{3}}\left\{q f^{\prime \prime}(q)+f^{\prime}(q)-f^{\prime}(q)^{2}\right\}
$$

The proofs of (5), (6) and (7) are in the Appendix.

\section{COMPUTER RESULTS}

Apparently, as it is illustrated by the computer runs (see Figure 1. and Figure 2.) for the critical case, the distribution of the life-cycle conditioned on ultimate extinction has 
a very long tail. The most significant result shown in the graphs is that for super- and subcritical branching models the conditional distributions of the lifetime given extinction coincide. The reason is explained in the next paragraph and an appropriate example is represented.

\section{DISCUSSION}

Inference from estimated total progeny or early extinction

One must be careful not to draw hasty conclusions after failed experiments. To be specific, suppose that three different types of bacterial culture $(\alpha, \beta, \gamma$, say) were introduced in similar wastewater and that each of these seems to have disappeared after some time, but that the $\alpha$-type strains were reported in highest numbers or over the largest period of time. Is it then most promising to bet on $\alpha$-type bacterial culture for a new experiment? The frequency of reports must be thought of as being positively correlated with the total progeny and the later with the reproduction mean of that bacterial culture. However such a conclusion would be erroneous, as we see in the following example. It is the number of times the process becomes extinct before it grows irreversibly, that will help to decide if the process is sub- or supercritical.

\section{Example}

Let $f_{p}(s)=p+0.35 s+(0.65-p) s^{2}$ be the p.g.f. of the reproduction law of a BellmanHarris process parametrized by $p=P$ \{the initial progenitor dies without any offspring $\}$. The case $p=0.325$ corresponds to the critical case $m=1,(0<p<0.325$ supercritical, 
$0.325<p \leq 0.65$ subcritical). Clearly, $q=q(p)$ is the smallest solution of $q=p+0.35 q+$ $(0.65-p) q^{2}$ in $[0,1]$ or $q=\min \left\{1, \frac{p}{(0.65-p)}\right\}$, and $m=m(p)=f_{p}^{\prime}(1)=1.65-2 p$, which decreases as $p$ increases, whereas

$$
E\left(N_{\infty} \mid T<\infty\right)=\frac{1}{1-f_{p}^{\prime}(q(p))}=\frac{1}{2|p-0.325|}
$$

which in the supercritical case increases as $p$ increases.

It is worth noting that within this family of p.g.f.'s, in the supercritical case $p<0.325$ the effect of conditioning on ultimate extinction is that $f(q s) / q$ is another member of the family with $p$ replaced by $0.65-p$. That is why if the supercritical process does die out then it is impossible from the statistical point of view to distinguish it from the subcritical one.

\section{CONCLUSION}

Extinction entails a very strong bias. If a decision maker decides to try again with that strains ( $\alpha$-type) which seems to have been best adapted so far he may exclude those strains with a much higher fertility rate $m$. The point is that he has to take that decision after extinction. It is simply very improbable that a process with a "comfortable" mean $m>1$ would die out late. The higher the mean of a population the more probable it becomes that this population would, after extinction, be excluded from further experiments.

We conclude that the problem is of a greater significance that it might appear at the first sight. Independent control studies to assess prior probability of extinction are likely to be environment-bias. 


\section{REFERENCES}

Athreya, K. and Ney, P., 1972. Branching Processes. Springer Verlag, Berlin, 287 pp.

Bhattacharjee, M. C., 1987. The time to extinction of branching processes and log-convexity: I. Probab. Eng. and Inf. Sci., I: 265-278.

Bruss, F. T. and Slavtchova-Bojkova, M., 1999. On waiting times to populate anenvironment and a question of statistical inference. J. Appl. Probab., 36: 261-267.

Foster, J. H., 1971. A limit theorems for a branching process with state-dependent immigration. Ann. Math. Stat., 42: 1773-1776.

Harris, T., 1989. The Theory of Branching Processes. Dover Publications Inc., New York, $230 \mathrm{pp}$.

Jacobson, M. E., 1985. Computation of Extinction Probabilities for the Bellman-Harris Branching Processes. Math. Biosci., 77: 173 - 177.

Jagers, P., 1975. Branching Processes with Biological Applications. Wiley, 268 pp.

Karlin, S. and Tavaré, S., 1982. Detecting particular genotypes in populations under nonrandom mating. Math. Biosci., 59:57-75.

Pakes, A. G., 1971. A branching processes with a state-dependent immigration component. Adv. Appl. Prob., 3: 301-314.

Pakes, A. G., 1975. Some results for non-supecritical Galton-Watson processes with immigration. Math. Biosci., 24: 71-92.

Powell, E. D., 1955. Some features of the generation times of individual bacteria, Biometrika, 42: 16-44.

Shaked, M. and Shantikumar, J. G., 1987. Characterization of some first passage times using log-concavity and log-convexity as ageing notions. Probab. Eng. and Inf. Sci., I: 
279-291.

Vatutin, V. A. and Zubkov, A. M., 1993. Branching Processes.Part II. Journal of Soviet Mathematics, Series: Probability Theory, Mathematical Statistics and Cybernetics., 67:3407-3485, Plenum Corporation. 